\title{
A Novel Framework for Analysing Antibiotic Resistance, Classifications and Treatment of Extended Spectrum Beta-Lactamases
}

\author{
Bader Saud Alotaibi", Jafar Ebrahim, Kadher Nivas and Farhan R. Khan \\ College of Applied Medical Sciences, Al-Quwayiyah Shaqra University, Kingdom of Saudi Arabia; \\ saudbadar2@gmail.com, jameela@su.edu.sa,knivas@su.edu.sa, riyazuddin@su.edu.sa
}

\begin{abstract}
Objectives: Identification of Beta-lactam clinically in laboratories is a necessary factor for the recognition of organisms which are antimicrobials resistant. The main aim of this study is to analyse the antibiotic resistance of Extended Spectrum Beta Lactamases (ESBLs) long with its classifications and its various treatments. Methods: Initially, the working principle or synthesis of antibiotics is elaborated using five modes such as the inference based on the cell wall, protein, nucleic acid, metabolic pathway and based on the cell membrane. Then, the antibiotic mechanism along with its types and various classifications are elaborated. Finally, various options for treating the infections which were caused by their ESBL causing bacteria are also identified. Findings: This study proposes a novel framework for analysing the resistance of antibiotic, classifications and the treatment necessary for the ESBLs in detail. Inferences based on the appearance of cell wall, protein, nucleic acid, metabolic pathway and the cell membrane provides the detailed architecture and working principle of the antibiotics. Classifications of ESBLs along with their types and from which bacteria it is derived from are also mentioned. Applications: This study focuses on the antibiotic characteristics ESBLs along with its various classifications, types and the treatments available for the infections occurred. This study can be further used for the identification of various treatment facilities for the infections caused by the ESBLs. Future research can be an analysis of more factors which influences the cause several associated health conditions due to these ESBLs
\end{abstract}

Keywords: Antibiotics, Extended-spectrum b-lactamases, Framework, Resistance, Synthesis

\section{Introduction}

Antibiotic resistance for supporting bacteria become an issue worldwide with significant results on diseases which are infectious in nature. The increased application and misapplication of antibiotics comprise major part to the existing condition. The resistance on antibiotics occurred in bacteria which causes infections. This can be classified based on the resistant for multidrug, resistant for methicillin, resistant for penicillin, resistant for vancomycin etc ${ }^{1}$. Antimicrobial agents such as the Beta-lactam depict particular procedure of treatment in case of infections caused by bacteria. Firm shield from the effects of bacteria on b-lactam and its combination has paved the way for continuous production of b-lactamases. This activity holds up the evolution of new antibiotics for b-lactam. This is called as ESBLs².
Diagnosis and consideration of these organisms which are drug resistant is an important task to resolve. Due to several conditions, it is difficult to solve the problems caused due to these ESBL generating organisms. Traces of various instances are registered because of these ESBL generating bacteria's which were reported by many researchers $\frac{3-7}{}$.

\section{Working Principle of Antibiotics}

The working principle or synthesis of antibiotics can be elaborated using five modes such as

- Cell wall inference.

- Protein and its inhabitation.

- Nucleic acid and its Interference.

*Author for correspondence 
- Metabolic pathway and its Inhibition.

- Cell membrane and disrupting its systematic order.

\subsection{Cell Wall Inference}

Antibiotics such as the penicillin and cephalosporin obstruct the enzymes which is accountable for the evolution of a layer called peptidoglycan which causes the cell wall inference synthesis. This is one among the five modes of antibiotic synthesis ${ }^{-}$.

\subsection{Protein and its Inhabitation}

One of the latest categories of antibiotics is the Oxazolidinones. These antibiotics interconnect with the synthesis of protein by the process of combining with the 30S, a distinct component of ribosome shown in Figure 1. Hence, it debilitates the ribosome. The class of antibiotics called Macrolides combines with the 50S, a distinct component of ribosomal subunit and thus it hamper the elaboration of the developing chains of polypeptides. Antibiotics also work by one of this process?

\subsection{Nucleic Acid and its Interference}

The interference of rifampicin, which is a class of medicines, belongs to anti-mycobacterials with a RNA polymerase which is DNA-directed. Antibiotics which intersect with the synthesis of protein can be categorised by their effect on procaryotic or euraryotic cells. It derives both of them either by combining or acting with the smaller or larger ribosome. By this process, the nucleic acid and its interference are synthesised.

\subsection{Metabolic Pathway and its Inhibition}

In this process, each and every block of the metabolic pathway is synthesised and the sulphonamides. By this process, the metabolic inhabitation is done.

\subsection{Cell Membrane and Disrupting its Systematic Order}

These processes done in the membrane called as cytoplasm which is present in the bacteria called as gram-positive. It is verbally assumed that the polymyxins employ their effect of preventing action by growing the membrane of bacteria in a state which leads to the extraction of contents present in the bacteria. Antibiotics show the fast activities of bacteria's by the process of combining it with the membrane of cytoplasm. It paves the way for a flow of potassium from the cell wall of bacteria which causes death to the particular cell ${ }^{8}$. The antibiotic activity is shown in Figure 1.

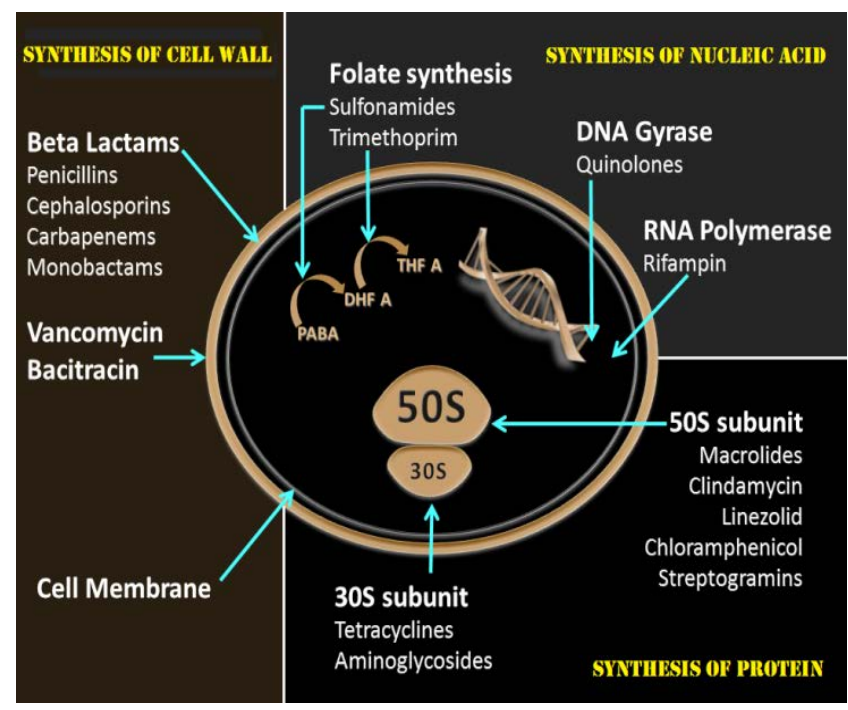

Figure 1. The mechanism of antibiotic activity ${ }^{13}$.

\section{The Mechanism of Antibiotic Resistance}

Resistance of antibiotic is the process of reducing the effects of an antibiotic drug given for the purpose of curing a disease. The effect of an antibiotic is assumed as failure of dosage when it not removed completely or hampers a bacteria or other microorganism which can cause diseases. If a pathogen acquires resistant more time to one drug or several drugs, then it is called as a multidrug resistance?

\section{ESBLs, Types and its Classifications}

This section depicts the definition of ESBLs, its types and various classifications based on its structure.

\subsection{Definition}

ESBLs are powerful against antibiotics based on betalactam for example ceftazidime, ceftriaxone etc. ESBLs are generated by tazobactam. ESBLs are found in Gramnegative bacteria. The most important beta-lactamase 
that is prevalent is TEM-1. Nearly 90 percentage of resistance from the ampicillin is present in the E.coli because of the TEM-1 in it. This can breakdown the penicillin. Hence, the TEM-1 is derived in to TEM-2, by changing the amino acids slightly.

Table 1. Type of the ESBLs and its derived from the bacteria

\begin{tabular}{|l|l|l|l|}
\hline No & Type & Name & Derived From \\
\hline 1 & Type 1 & SHV & Klebsiellaspp. \\
\hline 2 & Type 2 & TEM & E. coli \\
\hline 3 & Type 3 & CTX & Typhimurium \\
\hline 4 & Type 4 & OXA & P. aeruginosa \\
\hline 5 & Type 5 & PER & P. aeruginosa \\
\hline 6 & Type 6 & GES & Enterobacteriaceae \\
\hline
\end{tabular}

\subsection{Types and Classification}

The ESBLs can be classified based on three classes. It is proposed earlier in ${ }^{10}$. This is based on structure of the molecule. Base on their classification model, the beta-lactamase can be divided in to four schemes. The first scheme introduced by $\frac{11,12}{12}$. This class sub divides the ESBLs in to four sub groups such as A, B, C and D and also based on the similarity of protein present in enzymes. Table 1 depicts the Type of the ESBLs and its derived from the bacteria

\subsubsection{Group I (Class C) Beta-lactamases}

In this group of classification, chromosomes have lactamases such as clavolunate. Hence, when the bacteria are exposed to antibiotics increases the production of enzymes. When the antibiotic which belongs to the betalactame is more, then the production of beta-lactamase is increased in various levels.

\subsubsection{Group 2(Class A) Enzymes}

As the enzymes classified into two groups are docked by plasmid, they could easily be transmitted into different cells of bacteria's which generates a fast avoidance to these sorts of enzymes. The beta-lactamase and tazobactam inhibit the original group 2 enzymes. The main group 2 enzymes are TEM and SHV.

\subsubsection{Group 3 (Class B) Enzymes}

These are metallo-enzymes which destroys the carbapenems which are frequently present in Stenotrophomonas maltophilia. It is the Metallo-enzymes which break the carbapenems and other betalactams rather than monobactams.

\subsubsection{Group 4 (Class D) Beta-lactamases}

This belongs to the beta-lactamases consists of those unusual penicillinases which are not reserved towards the clavulanic acid. Four of these enzymes exhibit more mortality towards the breaking of carbenicillin. These show a unusual behaviour with respect to the inclusion of any metal. Hence, they represent another molecular class of beta-lactamase.

\section{Treatment of ESBLs}

Infections such as pneumonia which are caused by the ESBL producing E. coli where treated using older drugs. Drugs such as the Fosfomycin have a good effect for controlling the vitro activity against the ESBL-producing $E$. Coli or K. pneumoniae type of bacteria's. In Honk Kong, ESBL-producing bacteria's are considered to be more sensitive to Fosfomycin.Another treatment alternative is the Colistin which is used in the treatment of these types of micro-organisms. Although this antibiotic is small toxic, it is a final option that can be considered since there is no other option for these antibiotics.

\section{Conclusion and Future Enhancements}

Clinical identification of Beta-lactam in laboratories is an important factor for the recognition of organisms which are antimicrobials resistant. Resistance of antibiotic is the process of reducing the effects of an antibiotic drug given for the purpose of curing a disease. This study focuses on analysing the antibiotic resistance of the ESBLS along with the details classifications, types and from which bacteria it is derived. Analysis starts with the synthesis of antibiotics through five types of inference based on the arrangement of cell wall, protein nucleic acid, metabolic pathway and the architecture of cell membrane. Six types of ESBLs along with the details from which type of bacteria it is derived are also depicted. Further application of this analysis can be the identification of various treatment facilities for the infections caused by the ESBLs using the synthesis of inference in ESBLs. Future research can be an analysis of more factors which influences the cause several associated health conditions due to these ESBLs 


\section{References}

1. Department of Health UK Five Year Antimicrobial Resistance Strategy 2013 to 2018. Available from: https:// www.gov.uk/government/publications/uk-5-year-antimicrobial-resistance-strategy-2013-to-2018

2. Hannah BH. Antimicrobial-resistant Escherichia coli from environmental waters in Northern Colorado. Journal of Environmental and Public Health. 2019; 13. https://doi.org/10.1155/2019/3862949 PMid:30906330 PMCid:PMC6397973

3. Pitout JD, Laupland KB. Extended-spectrum b-lactamase producing enterobacteriaceae: an emerging public health concern. The Lancet Infectious Diseases. 2008; 8(3):159-66. https://doi.org/10.1016/S1473-3099(08)70041-0

4. Rupinder B, Geeta W, Shikha J. Prevalence of extended spectrum b-lactamases in multidrug resistant strains of gram negative Bacilli. Journal of Academia and Industrial Research. 2013; 1(9):558-60.

5. Peirano G, Bradford PA, Kazmierczak KM. Global incidence of carbapenemase-producing Escherichia coli. Emerging Infectious Diseases. 2014; 20:1928-31. https://doi.org/10.3201/eid2011.141388 PMid:25340464 PMCid:PMC4214325

6. Vila J, Saez-Lopez E, Retal J. Escherichia coli: an old friend with new tidings. FEMS Microbiology Reviewa. 2016; 40:437-63. https://doi.org/10.1093/femsre/fuw005 PMid:28201713

7. Benton B, Breukink E, Visscher I, Debabov D, Lunde C, Janc J, Mammen M, Humphrey P. Telavancin inhibits peptidoglycan biosynthesis through preferential targeting of transglycosylation: evidence for a multivalent interac- tion between telavancin and lipid II. International Journal of Antimicrobial Agents. 2007; 29:51-2. https://doi. org/10.1016/S0924-8579(07)70166-8

8. Öztürk H, Ozkirimli E, Ozgur. A Classification of beta-lactamases and penicillin binding proteins using ligand-centric network models. Plos One. 2015; 10 (2). https://doi.org/10.1371/journal.pone.0117874 PMid:25689853 PMCid:PMC4331424

9. Viloria A, Urbina MC, Rodríguez LG, Muñoz Ap. Predicting of behavior of Escherichia coli Resistance to imipenem and meropenem, using a simple mathematical model regression. Indian Journal of Science and Technology. 2016; 9(46):1-5. https://doi.org/10.17485/ijst/2016/v9i46/107379

10. Bush K, Jacoby GA, Medeiros AA. A functional classification scheme for beta-lactamases and its correlation with molecular structure. Antimicrobial Agents and Chemotherapy. 1995; 9:1211-33. https://doi.org/10.1128/ AAC.39.6.1211 PMid:7574506

11. Ambler RP. The structure of b-lactamases. Philosophical Transactions of the Royal Society B: Biological. 1980; 289:321-31. https://doi.org/10.1098/rstb.1980.0049 PMid:6109327

12. Bonnet R. Growing group of extended-spectrum betalactamases: the CTX-M enzymes. Antimicrob Agents Chemother. 2004; 48:1-14. https://doi.org/10.1128/ AAC.48.1.1-14.2004 PMid:14693512 PMCid:PMC310187

13. Li F, Collins JG, Keene FR. Ruthenium complexes as antimicrobial agents. Chem Soc Rev. 2015 Apr 21; 44(8):2529-42. Available from: https://pubs.rsc.org/en/content/articlehtml/2015/cs/c4cs00343h https://doi.org/10.1039/ C4CS00343H PMid:25724019 